\title{
Conceptual Structures Represented by Conceptual Graphs and Formal Concept Analysis
}

\author{
Guy Mineau ${ }^{1}$, Gerd Stumme ${ }^{2}$, Rudolf Wille ${ }^{2}$ \\ 1 Département d'Informatique, Faculté des Sciences et de Génie, Université Laval, \\ Cité universitaire, Québec, Canada, G1K 7P4; mineau@ift.ulaval.ca \\ 2 Technische Universität Darmstadt, Fachbereich Mathematik, D-64289 Darmstadt, \\ Germany; \{stumme, wille\}@mathematik.tu-darmstadt.de
}

(C) Springer-Verlag Berlin-Heidelberg 1999

\begin{abstract}
Conceptual Graphs and Formal Concept Analysis have in common basic concerns: the focus on conceptual structures, the use of diagrams for supporting communication, the orientation by Peirce's Pragmatism, and the aim of representing and processing knowledge. These concerns open rich possibilities of interplay and integration. We discuss the philosophical foundations of both disciplines, and analyze their specific qualities. Based on this analysis, we discuss some possible approaches of interplay and integration.
\end{abstract}

\section{Conceptual Structures in Knowledge Representation}

Conceptual structures in knowledge representation are models (or artifacts) representing a perceived reality. With computer applications, these models support and delimit the kind of processing that a knowledge system will be able to carry out. Hence, the semantics they carry is of the outmost importance to the soundness and completeness of the applications that use them. With Artificial Intelligence related applications, these models represent human knowledge in a format available to an inference engine. To describe knowledge, a multitude of knowledge representation formalisms were devised. Among them, semantic networks aim at bringing together knowledge representation and graphical formalisms, hoping to alleviate the knowledge modeling and transfer problems pertaining to knowledge acquisition.

Through the years, different semantic network based formalisms were introduced, each having a different scope. Some dealt with linguistic knowledge, using the assumption that knowledge is necessarily expressible using natural language (cf. [33]). One of the underlying reasons for using this assumption was to facilitate the interfacing between the knowledge system and both the knowledge engineer and the end-user, since both could use a natural language based interface to communicate with the system. Furthermore, this would improve the interpretability of the achievements of the system at any given time.

Some other formalisms had preoccupations pertaining to complexity, as their applicability was often challenged by complexity results which discarded them 
from the spectrum of formalisms usable for the development of large-size applications (cf. [9], [23]). Applicability considerations must often restrict the kind of knowledge that can be represented by the formalism; it is referred to as the completeness/tractability trade-off. For instance, modal quantification, though very useful for many applications such as planning, is often omitted in order to simplify inference mechanisms.

Further formalisms put their emphasis on some logical aspects such as quantification and scope in order to address problems that require them (see [59]). The motivation behind this approach is to allow simple modeling of complex interrelations between sets of individuals. In brief, the nature of the knowledge captured by these formalisms varies greatly depending on the scope of the formalism: the same knowledge could be represented differently using different formalisms.

Twenty years ago, Ron Brachman surveyed the most popular semantic network based formalisms and identified five levels pertaining to knowledge representation [7]:

- Implementational Level: The primitives are nodes and links where links are merely pointers and nodes are simply destinations for links. On this level there are only data structures out of which to build logical forms.

- Logical Level: The primitives are logical predicates, operators, and propositions together with a structured index over those primitives. On this level logical adequacy is responsible for meaningfully factoring knowledge.

- Epistemological Level: The primitives are conceptual units, conceptual subpieces, inheritance and structuring relations. On this level conceptual units are determined in their inherent structure and their interrelationships.

- Conceptual Level: The primitives are word-senses and case relations, objectand action-types. On this level small sets of language-independent conceptual elements and relationships are fixed from which all expressible concepts can be constructed.

- Linguistic Level: The primitives are arbitrary concepts, words, and expressions. On this level the primitives are language-dependent, and are expected to change in meaning as the network grows.

Brachman discusses general criteria for judging the utility and formality of a given network language with respect to the described levels, namely neutrality, adequacy, and semantics. In a network, each level should be neutral (i.e.: not forcing any choice), and adequate in its support, to the level above it, and should have as definite as possible a well-defined semantics.

More recently, Randall Davis, Howard Schrobe, and Peter Szolovits stated five basic principles about knowledge representation in their critical review and analysis of the state of the art in knowledge representation [10]: A knowledge representation is (1) a surrogate, (2) a set of ontological commitments, (3) a fragmentary theory of intelligent reasoning, (4) a medium for efficient computation, and (5) a medium of human expression. These principles together with Brachman's level description and criteria for semantic networks shall be used as 
a framework for discussing the role of conceptual structures in knowledge representation performed by Conceptual Graphs and Formal Concept Analysis. The main aim of this paper is to investigate possibilities of interplay and integration of Conceptual Graphs and Formal Concept Analysis. For preparing this, we first give a brief introduction to both disciplines via illustrating examples in Section 2. Then their philosophical foundations are outlined in Section 3. In Section 4, methods of knowledge representation are described and evaluated, so that, finally in Section 5, possibilities of interplay and integration can be discussed.

\section{Conceptual Graphs and Formal Concept Analysis: Illustrating Examples}

To make this paper as much as possible self-contained, we give in this section brief introductions to Conceptual Graphs and Formal Concept Analysis via some illustrating examples. The reader who is familiar with those disciplines is recommended to skip the corresponding subsections, respectively.

\subsection{Conceptual Graphs}

Conceptual Graphs are a knowledge representation mechanism together with a reasoning mechanism. A conceptual graph is a labeled graph that represents the literal meaning of a sentence or even a longer text. It shows the concepts (represented by boxes) and the relations between them (represented by circles) (cf. [38]). As illustrating example, we show how the following text of instructions for decalcifying a coffee machine [1, p.32] may be represented by a conceptual graph:

In order to decalcify a coffee machine in an environment friendly way, one must fill it up with water and put in two teaspoons of citric acid (from the drugstore). Then one must turn it on and let the mixture go through the machine. Then, one must fill it up with clear water and let it go through the machine, twice.

Figure 1 shows a conceptual graph that represents the text and makes the text with its instructions tractable by a computer. For instance, it could be part of a document retrieval system for technical instructions. All concepts represented in the graph are generic concepts, because the text does not describe a specific situation, but instructions which are applicable to all kinds of coffee machines. The concept types (e.g., WATER) of conceptual graphs are usually organized in a type hierarchy. In this example, the concept types are all incomparable (with the exception ACTION < PROCESS). For a document retrieval system with many texts, however, the resulting type hierarchy will become larger and, as the texts treat similar topics, there will also arise comparable concept types. For keeping our introduction short, we consider all concept types as primitive types, i.e., we do not use type definitions. 


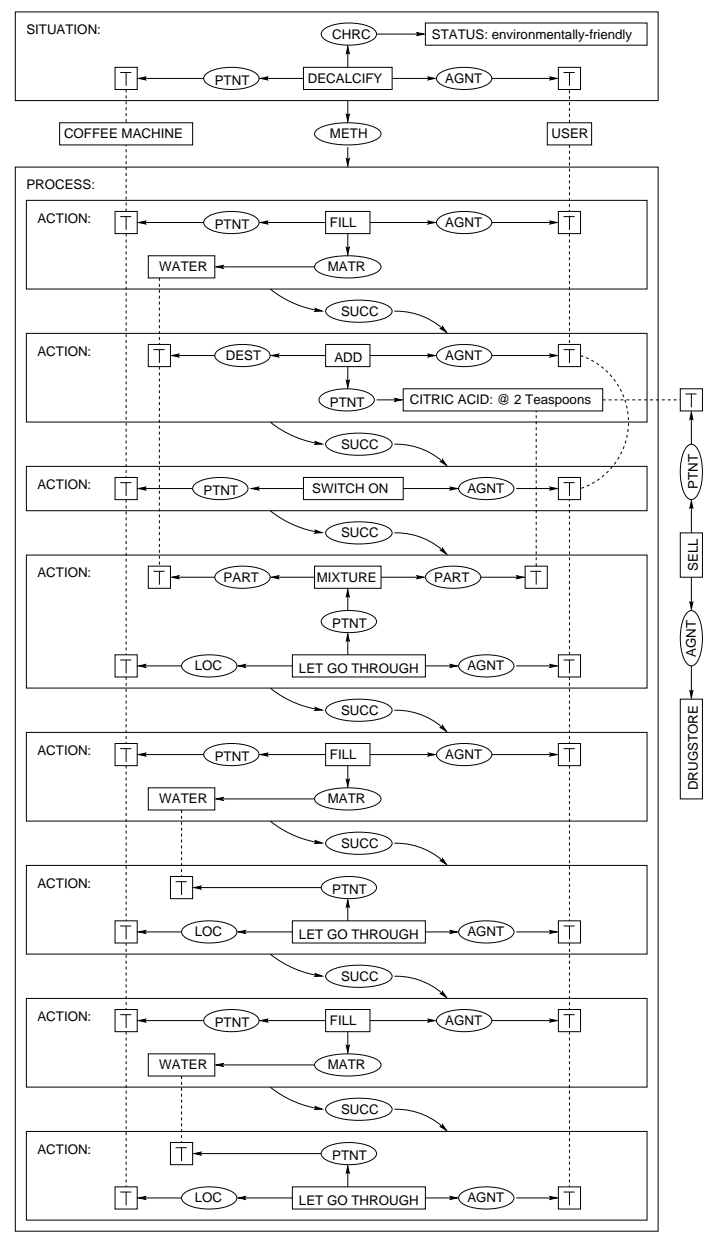

Fig. 1. A conceptual graph describing how to decalcify a coffee machine

In the conceptual graph, the process of decalcifying a coffee machine is splitted into succeeding actions. These actions are represented by larger boxes, called contexts, linked by the (SUCC)-relation. The sequel of all these actions is grouped together to a process which is stated as a method for decalcifying a coffeemachine in an environmentally-friendly way. Observe how the identity of the user and the coffee-machine is maintained throughout the whole process by dotted lines, called coreference links.

The conceptual graph in Figure 1 represents only the literal meaning of the instructions. It does not include background knowledge of a domain expert which is not provided by the text. For a document retrieval system, this approach is sufficient, because we assume that the end-user is familiar with the basic 


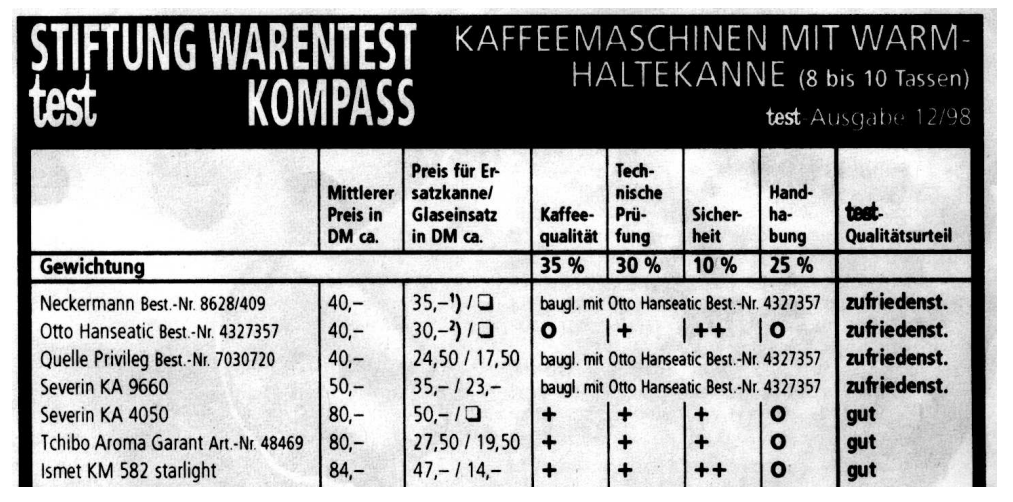

Fig. 2. Part of a test report about coffee machines with integrated thermos flasks.

concepts of the domain. If there is the need of additional explanations, the set of documents must be extended.

This example shows that the decision to which extent domain knowledge should be formalized depends on the purpose. For a system with a human enduser one can rely on his background and common sense knowledge, while, for a knowledge base for a robot system which automatically decalcifies coffee machines, much more of the background knowledge has to be made explicit. For instance, we must let the system know that no coffee machine can be clean and dirty at the same time.

\subsection{Formal Concept Analysis}

Formal Concept Analysis is mainly used for analyzing data tables. We demonstrate this by a typical example: an investigation of coffee machines with integrated thermos flasks. Figure 2 shows part of a test report ([48]). This data table represents a so-called many-valued context in the sense of Formal Concept Analysis. ${ }^{1}$ In order to obtain a concept lattice for investigating the data, we have to derive a one-valued context. Therefore we must decide which of the attributes are important, and how they shall be translated into one-valued attributes. The result of this so-called (plain) conceptual scaling is the formal context in Figure 3 . The first four machines in Figure 2 are constructively indentical, so they are all represented by 'Otto Hanseatic' in the formal context. We emphasize that the choice of the conceptual scales depend on the purpose of the analysis. For instance, other attributes may be suitable for market analysis than for decision support. The attributes in Fig. 3 have been chosen in order to support a buyer in choosing a coffee machine.

\footnotetext{
${ }^{1}$ For the basic definitions of Formal Concept Analysis, see for instance [14] or [53].
} 


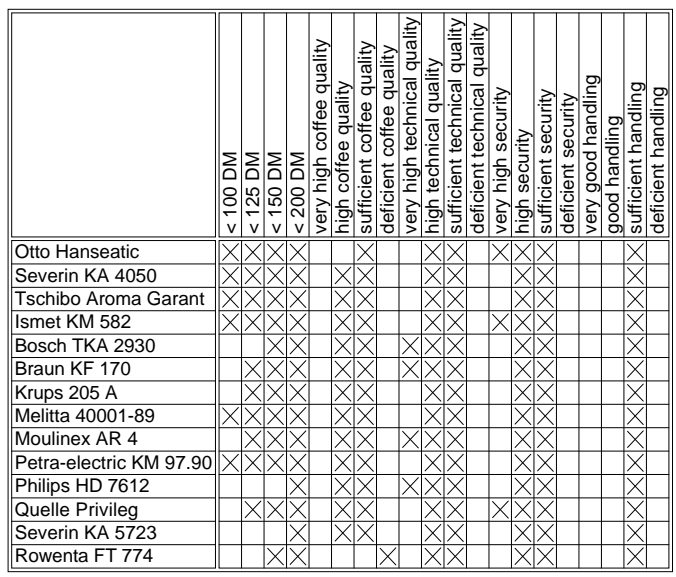

Fig. 3. Formal context derived from the table in Figure 2

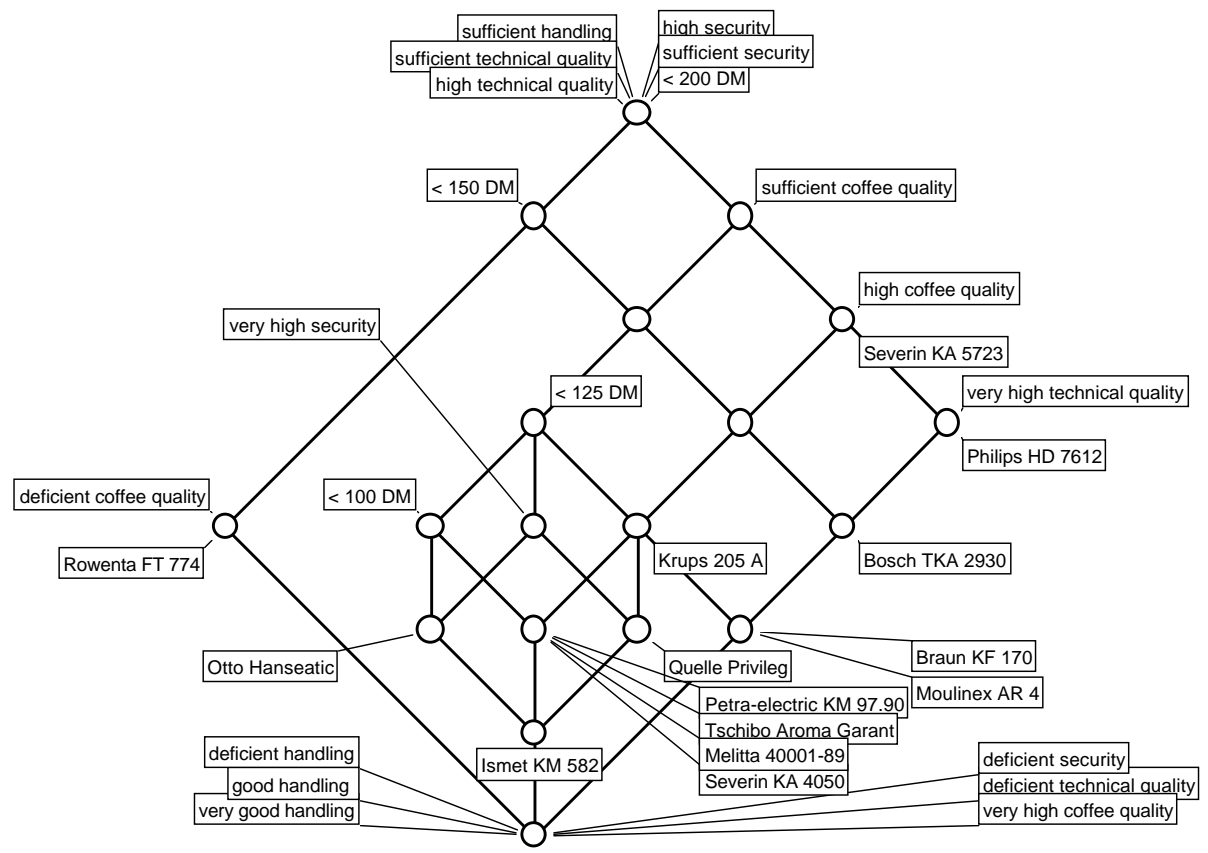

Fig. 4. The concept lattice derived from the formal context in Fig. 3

From the formal context, the concept lattice is computed, and visualized by a line diagram (cf. Fig. 4). We recall that each circle in the diagram represents a formal concept, i.e., a pair $(A, B)$ where $A$ is a subset of the set of objects, $B$ 
a subset of the set of attributes, and both are maximal such that each object has each attribute and vice versa. The extent $A$ of a concept contains all objects whose labels are attached at concepts which are lower in the diagram, and the intent $B$ contains all attributes whose labels are attached at concepts which are higher in the hierarchy. For instance, the rightmost concept has the objects 'Philips HD 7612', Bosch TKA 2930', 'Braun KF 170', and 'Moulinex AR 4' in its extent, and the attributes 'very high technical quality', 'high coffee quality', sufficient coffee quality', 'sufficient handling', ... ' '<200 DM' in its intent.

In the diagram, we can see that all machines have at least 'sufficient handling', 'high technical quality', 'high security', and cost ' $<200 \mathrm{DM}$ '. There is only one machine producing deficient coffee quality, 'Rowenta FT 774', and it does not even belong to the cheaper machines. On the other hand, 'Ismet KM 582', 'Braun KF 170', and 'Moulinex AR 4' have most of the positive attributes. Here, one has to decide between 'very high security' and '< 100 DM' on one side and 'very high technical quality' on the other. The fact that the label 'very high technical quality' is below the label 'high coffee quality' indicates that for all tested coffee machines the implication 'very high technical quality' $\rightarrow$ 'high coffee quality' holds.

In many applications, it is an advantage to limit the represented knowledge. Then the whole information can be visualized by one line diagram, which supports communication between knowledge engineers, experts, and novices. For instance, line diagrams of concept lattices have been used to a large extent for visualizing repertory grids tests in the treatment of patients suffering from bulimia ([41]). The diagrams were small enough to be understood by the patients, and at the same time they provided all information provided by the repertory grid. A similar application was the visualization of the water pollution of Lake Ontario for decision support ([42]).

For larger data, the attributes of the derived context are grouped to conceptual scales and, for each scale, its concept lattice is determined. By combining the concept lattices in nested line diagrams, information about the interplay of attributes is provided. Such applications are supported by the management system TOSCANA for Conceptual Information Systems. Examples of Conceptual Information Systems have been presented at ICCS'96 and ICCS '98 ([44], [15]). Other applications are, for instance, a conceptual information system about the medical nomenclature system SNOMED ([32]), a system for investigating international cooperations ([19]), and a retrieval system for a library ([20], [31]). The largest implemented system has 600,000 objects, and a system with 20,000,000 objects is planned for a basket analysis of a credit card company.

\section{Philosophical Foundations}

Conceptual Graphs and Formal Concept Analysis are each based on an elaborated philosophical understanding leading its development and application. For investigating the possibilities of their interplay and integration, it is necessary to bring the philosophical bases of both disciplines to mind. At first glance the 
philosophical foundations of Conceptual Graphs and Formal Concept Analysis seem to widely coincide: They both have conceptual structures as central theme of inquiry, both emphasize graphical representations for activating human thinking, both rely on Peirce's Pragmatism, and both aim to represent and process knowledge. But, since they were grown out of different roots and purposes in different scientific communities, each of the philosophical foundations has its special quality. This shall be outlined in this section to understand better how Conceptual Graphs and Formal Concept Analysis may be successfully combined.

According to [34], conceptual graphs arose in the Artificial Intelligence community when John Sowa started in 1968 to combine semantic networks and linguistic dependency graphs for forming a semantic representation of natural language. The used box and circle notation was influenced by the plastic templates used for computer flow charts. A systematic presentation of conceptual graphs which adapted also ideas of relational database theory was first published in [36]. Conceptual Graphs matured to a precise system of logic when John Sowa accommodated a new foundation based on Peirce's Logic of Existential Graphs in 1983. With this foundation, Sowa could present Conceptual Graphs as a rich and matured discipline in his book "Conceptual Structures: Information Processing in Mind and Machine" [37].

In this book Sowa views Conceptual Graphs as a knowledge representation language based on Linguistics, Psychology, and Philosophy, and in his "Conceptual Graphs Summary" [38] as a system of logic based on the existential graphs of Charles Sanders Peirce and the semantic networks of Artificial Intelligence. The key words "knowledge" and "logic" refer to a philosophical tradition of over two and a half millennia. Sowa extensively discusses this tradition in his new book "Knowledge Representation: Logical, Philosophical, and Computational Foundations" [39] to clarify the ontological basis of conceptual graphs. Starting with Heraclitus and Plato, he explains the systems of categories proposed by Aristotle, Kant, Peirce, Husserl, Whitehead, and Heidegger for deriving a new system of twelve categories which are obtained by associating the three major distinctions: Thing - Relation - Mediation; Physical - Abstract; Continuant Occurrant. The categories provide a framework for classifying everything that exists, and can therefore be considered as a philosophical basis for hierarchies of concept types that are assumed for conceptual graphs.

In [39], the philosophical tradition for understanding the logical character of conceptual graphs is presented too. In sketching the history of logic, Sowa discusses Aristotle's syllogisms and their scholastic classification, first attempts toward automated reasoning by Lull and Leibniz, and the first major contributions to modern mathematical logic by Boole, Frege, Peirce, Schröder, Peano, Russell, and Whitehead. In the same way logic became more mathematical, the connections to common sense and natural language got weaker. Against this trend, conceptual graphs were designed as a logic with a human readable notation and a semantic basis of natural language for representing models of the real world or other possible worlds. In [37], Sowa describes this as follows:

- Rules of syntax map the graph to and from sentences in natural languages. 
- Arcs of the graphs correspond to the function words and case relations of natural language.

- Nodes of the graphs are intensional concepts of individuals that may exist in the real world or some hypothetical world.

- Exact reasoning is based on Peirce's existential graphs [...]. The graphs are a complete notation for first-order logic with direct extensions to modal and higher-order logic.

- Plausible reasoning is based on schemata and prototypes, which codify the defaults and family resemblances that accommodate the variability of the real world.

- Model theory uses the same kinds of graph structures for both formal models and propositions about models.

Conceptual graphs have the ontological status of a model: they simulate significant structures and events in a possible world; a set of axioms, called "laws of the world", must at all times be true of the graphs; and certain graphs, called "schemata" and "prototypes", serve as patterns or frames that are joined to form the model. Understanding the knowledge of something as the ability to form a mental model that accurately represent the thing as well as the actions that can be performed by it and on it, it becomes clear why conceptual graphs as mental models are successful in knowledge representation, acquisition, and processing.

Formal Concept Analysis arose in the Fachbereich Mathematik of the TH Darmstadt out of efforts toward a better understanding of the relationships of mathematics to the world. These efforts led to the program of restructuring mathematics, an activity of reworking mathematical developments in order to integrate and to rationalize origins, connections, interpretations, and applications (cf. [54]). Besides several other attempts to restructure mathematical disciplines, the restructuring of mathematical order theory and lattice theory was successfully approached (see [49], [25]). The most fruitful result of this restructuring was the interpretation of complete lattices as concept lattices, which increasingly opened connections to new fields of applications. In this way, Formal Concept Analysis became established as a discipline of applied mathematics, based on a mathematization of concept and conceptual hierarchy, which activates mathematical thinking for conceptual data analysis and knowledge processing (see [14]).

Formal Concept Analysis is grounded on the philosophical understanding of a concept as a unit of thought constituted by its extension and its intension. This concept understanding has grown during centuries from Greek philosophy to late Scholastic and has finally found its modern formulation in the 17 th century by the Logic of Port Royal (cf. [3], [16], [52]). Its actual use is exemplified by the German Standards DIN 2330 "Begriffe und Benennungen" [11]. Logically, Formal Concept Analysis fits in the tradition of the classical philosophical logic with its elementary doctrines of concepts, judgments, and conclusions (cf. [18], [54], [27]).

The mathematical notions of formal context, formal concept, and concept 
lattice - resulting from the mathematization of context, concept, and conceptual hierarchy - are not understood as realistic models, but as artifacts which may support human thinking, communication, and argumentation. Why such a support is possible, may be explained on the ground of Heidegger's characterization of Modern Science as guided by "das Mathematische" [17]. Martin Heidegger understands "das Mathematische" as the formal conception in which we take note of realities and acquire their use. Mathematics is then viewed as a specific culture of thinking in which "das Mathematische" is elaborated and conventionalized, caused by a complex process of communication within the respective community of mathematicians (cf. [58]). This explains that mathematics may support human thinking in general because mathematics is arising out of "das Mathematische" in human thought. The mathematizations used in Formal Concept Analysis are therefore understood as purpose-oriented constructions shaping "das Mathematische" in the thoughts which are mathematized.

As mathematical discipline, Formal Concept Analysis is semantically based on set theory as used in today's mathematics. On this basis, all its theoretical notions and results are of mathematical nature and have first of all to satisfy mathematical standards of adequacy and correctness. But as part of applied mathematics, Formal Concept Analysis also activates systematically connections of the mathematical theory to reality. Concerning such connections, human communication and argumentation must be considered since mathematical methods cannot grasp realities without an eventually serious loss of content. Therefore mathematizations have always to keep connections to their origins so that the consequences of mathematical treatments may be rationally analysed and interpreted in human communication. One way of establishing ties between mathematical theory and reality is to insert pieces of natural language into mathematical structures and their graphical representations. Formal Concept Analysis does this, for instance, by keeping the namings of objects and attributes and by using them in lattice diagrams to give conceptual meaning to structural relationships.

According to Peirce's Pragmatism, human knowledge is always incomplete and continuously requires intersubjective communication and argumentation for its formation. This understanding of knowledge is basic for Formal Concept Analysis (cf. [51]). Therefore its formalizations for the structural and computational treatment of data and knowledge are performed in such a way that they may support human communication and argumentation for establishing intersubjectively assured knowledge. For connecting all aspects of contents and formal settings, there is the vision of formally developing "conceptual landscapes of knowledge" which allow humans to commonly explore and acquire knowledge satisfying their specific requests (see [56]).

Although the above description shows quite different philosophical foundations, Conceptual Graphs and Formal Concept Analysis could join their specific qualities for obtaining a broader spectrum of methods of representing and processing conceptual structures. For instance, the world semantics of conceptual graphs and the set semantics of concept lattices could complement each other to increase the meaningfulness of representations and the mathematical tractability 
of structures; this would include an appropriate use of linguistic contexts and formal contexts for specific purposes in knowledge representation. More generally, Conceptual Graphs and Formal Concept Analysis could join to merge different developments of logics such as philosophical logics, mathematical logics, description logics, situational logics and others.

\section{Conceptual Graphs and Formal Concept Analysis: Methods of Representation}

The five levels of Brachman provide a framework for discussing the role of Conceptual Graphs and Formal Concept Analysis in knowledge representation. In the disussion, we also refer to the five principles of Davis, Schrobe, and Szolovits.

Implementational Level. The basic data structures of Conceptual Graphs are graphs in the usual mathematical understanding, as Sowa points out in [38]: "The dyadic relation LINK is the only primitive in the formal theory. All other conceptual relations may be defined in terms of it." However, virtually, all research in the Conceptual Graph community is done on the higher levels of representation. In Formal Concept Analysis, the basic data structures are formal (dyadic) contexts formed by a binary relation and many-valued (triadic) contexts formed by a ternary relation. The simplicity of these data structures makes clear that they are not understood as models imaging reality, but as surrogates or artifacts. On the implementational level, "there is nothing inherently "semantic"" [7]; indeed, graphs and formal contexts are abstract mathematical structures without any content. Implementational issues of Formal Concept Analysis are discussed in [47].

Logical Level. In Conceptual Graphs and in Formal Concept Analysis, the logical level is activated in processing of the basic data structures. For Conceptual Graphs the logical primitives are the (abstract) concept and relation types and the connectives representing conjunctions, references, coreferences, contexts, actors, and type definitions. Reasoning is a major activity in processing Conceptual Graphs. Graph transformation rules (like projections) provide a fragmentary theory of intelligent reasoning which is inherent to Conceptual Graphs. Also the Peircing rules of deduction based on the context mechanism is also typical of the system of logic that the conceptual graph theory is. By mapping Conceptual Graphs to First Order Logic by the $\Phi$-operator, other reasoning mechanisms can be activated for efficient computation as well. In Formal Concept Analysis, the (formal) object, attribute, and concept names are logical primitives which allow the composition of further predicates by logical connectives and quantifiers (cf. [5], [28]). Then the means for logical reasoning are taken from Predicate Logic. As logical operators, the numerous context constructions for one- and many-valued contexts may be considered; the mostly used context construction is the semiproduct which is basic for 'plain conceptual scaling' (described in Section 2.2). 
Epistemological Level. The epistemological level addresses "the possibility of organizations of conceptual knowledge into units more structured than simple nodes and links or predicates and propositions, and the possibility of processing over larger units than single network links. The predominant use of concepts as intensional descriptions of objects hints that there is a class of relationship that is not accounted for by the [other] four levels [...]. This kind of relationship relates the parts of an intension to the intension as whole, and one intension to another" [7]. Brachman observed that for the semantic network representations of that time, this epistemological level is missing.

For Conceptual Graphs, the epistemological level is still not fully activated. The structures of type hierarchies belong to this level and also the expansion and contraction operators which are defined in the Conceptual Graph theory (see [37]). These operators allow multiple representations of the same knowledge because they provide a mechanism for the aggregation of lower-level constructs into higher abstractions (used at the conceptual level). Based on lambda expressions, these operators allow the representation and processing of the same knowledge at different levels of granularity. Type definitions implement these lambda expression definitions. They are solely based on extensional semantics and, in our opinion, they may not be sufficient to provide a sound justification for their existence. Beside representing the internal structure of concepts, another feature of the epistemological level is the representation of 'inheritance', which, according to [7], "is not a logical primitive; on the other hand, it is a mechanism assumed by all conceptual level nets [in particular Conceptual Graphs], but not accounted for as a 'semantic' (deep case) relation."

Concept lattices, the core structures of Formal Concept Analysis, are located on the epistemological level: Formal concepts are considered as "formal objects, with predetermined internal organization that is more sophisticated than sets of cases" [7]. Formal concepts bring together extensional and intensional views on 'concepts', and represent explicitly inheritance by refering to the set semantics of the intents (or extents) of the formal concepts. Concept lattice constructions also belong to the epistemological level. As Formal Concept Analysis is founded on lattice theory, lattice constructions and lattice decompositions can be activated for establishing more complex concept hierarchies out of simpler ones, and, vice versa, for reducing complex concept hierarchies to simpler ones. Constructions like direct products and tensor products of concept lattices and decompositions like subdirect and atlas decompositions have been successfully applied in data analysis. It is interesting to note that most concept lattice constructions (decompositions) have as counterpart a context construction on the logical level (see [14]). As formal contexts are only 'logarithmic in size' compared to the concept lattice, the knowledge representation on the logical level can be seen in the light of the fourthprinciple of Davis, Schrobe, and Szolovits as a medium of efficient computation.

The interplay between representations by formal contexts and by concept lattices supports also the fifth principle: The acceptance of Formal Concept Analysis as a medium of human expression by users from various domains (consider 
for instance the patients mentioned in Section 2.2 who have no higher mathematical education) results from the visualization of the data (as present on the logical level) on the epistemological level. In particular, the construction of complex lattices as (sub-)direct product of simpler lattices allows the visualization of complex data by nested line diagrams. The corresponding construction on the logical level is the semiproduct of conceptual scales, which is applied for efficiently querying the database.

Conceptual Level. The conceptual level is the major domain of Conceptual Graphs, just as it is for most semantical networks. Concept meanings are structured in type hierarchies according to levels of generality. They are linked by primitive semantic relations understood as those relations "that the verb in a sentence has with its subject, object, and prepositional phrase arguments in addition to those that underlie common lexical, classificational, and modificational relations" [35]. According to [7], "networks on this level can be characterized as having a small set of language-independent conceptual elements (namely, primitive object- and action-types) and conceptually primitive relationships (i.e., 'deep cases') out of which all expressible concepts can be constructed". In the theory of Conceptual Graphs, there is no predefined relation type other than LINK, which is the most general relation, being already formally located on the logical level. By applying concept expansion as described on the epistemological level, each concept type can be expanded such that all relationships are represented by the LINK relation. Hence case grammar based relations are in no way compulsory to the development of Conceptual Graph systems. However, the way Conceptual Graphs are used in practice identifies them clearly as being on the conceptual level: Although the LINK-relation is the only primitive relation in theory, in applications, the starter set of relation types is used as if consisting of primitive types in practice. The starter set plays the role of the small set of language-independent conceptually primitive relationships mentioned by Brachman. We conclude that the typical use of relation types identifies Conceptual Graphs as a knowledge representation formalism which is located mainly on the conceptual level.

In Formal Concept Analysis, word-senses are represented by the context attributes which lead to a contextual representation of concept intensions. As primitive case relations, there are defined four basic relations: an object has an attribute, an object belongs to a concept, a concept abstracts to an attribute, and a concept is a subconcept of another concept. These four relations are used as primitive relations in the mathematical model for Conceptual Knowledge Systems described in [22]. Other relations (especially meronomy) are discussed in $[30]$.

Linguistical Level. Neither Conceptual Graphs nor Formal Concept Analysis provide (at least not in their core) language-specific primitives. The linguistical level is activated only indirectly. For Formal Concept Analysis, this activation is discussed in more detail in [2] from a pragmatic-semiotic point of view. 
There the existence of qualified knowledge [anspruchsvolles Wissen] is claimed to depend from the existence of a community of discourse in which the knowledge is intersubjectively constituted.

Considering that the vocabulary (made of aggregations and primitive types) used to describe all Conceptual Graphs in a system is highly dependent on the type definition mechanism, we feel that Conceptual Graphs need to consider some complementary mechanism to ensure some epistemological soundness of the type definition mechanism. As Formal Concept Analysis is strong on the epistemological level, we are confident that Formal Concept Analysis can contribute to this. On the other hand, the experience of data analysis with Formal Concept Analysis has shown that there are applications with a need to enhance the expressiveness of Formal Concept Analysis on the conceptual level. We expect from the interplay of both theories a step in this direction.

\section{$5 \quad$ Interplay and Integration}

As already stated in Section 3, Conceptual Graphs and Formal Concept Analysis have basic concerns in common: the focus on conceptual structures, the use of diagrams for supporting communication, the orientation by Peirce's Pragmatism, and the aim of representing and processing knowledge. These concerns open rich possibilities of interplay and integration. Since both disciplines have their specific quality besides their common concerns, they may also complement each other. In this section, we discuss possible approaches of interplay and integration; but, because of the lack of space, we have to restrict to two themes: conceptual hierarchies and systems of logic.

\subsection{Interplay: Deriving and Improving Conceptual Hierarchies}

Conceptual hierarchies are basic in Conceptual Graphs and Formal Concept Analysis where they are mainly considered at the epistemological and the conceptual level. For conceptual graphs hierarchies of concept types are presumed as an ontological commitment. Often they are taken from conceptual taxonomies which, in general, are only ordered sets. But, for many purposes, it is desirable that the concept types form even a lattice. This can be effectively derived by methods of Formal Concept Analysis. In [13], Bernhard Ganter and Sergei Kuznetsov describe simple algorithms with satisfactory complexity bounds for minimally completing a finite ordered set to a lattice. For obtaining a meaningful type lattice, the minimal extension of a given ordered set of concept types might not be satisfactory. For finding useful completions, the construction of concept lattices of meaningfully deduced formal contexts can be offered as general method. The formal context need not contain all objects and attributes that belong to the concepts of the type hierarchy; it is sufficient to have enough typical objects and attributes. Then its concept lattice can be used as completed type hierarchy. The formal context can either be derived from an existing data set 
(if it provides enough typical objects and attributes), or it must be interactively acquired from some human expert.

For instance, there is a concept type COFFEE MACHINE in the type hierarchy for the document retrieval system in Section 2.1. This concept has in its extension all existing coffee machines and in its intension all attributes common to all coffee machines. In the knowledge acquisition process, however, it is sufficient to ask the user only for some few objects and attributes which distinguish this concept from the other involved concepts such as USER or CITRIC ACID.

Formal Concept Analysis provides different knowledge acquisition algorithms which can be used for that purpose: Attribute Exploration [14], Distributive Concept Exploration ([43]), and Concept Exploration ([46]). In [4], Franz Baader has used Attribute Exploration together with the Subsumption Algorithm of Description Logics for automatically deriving a $\bigwedge$-completion for an arbitrarily given type hierarchy. For a general overview concerning knowledge acquisition by methods of Formal Concept Analysis, see [50] and [45].

Methods of Formal Concept Analysis may also be applied to the generalization order on conceptual graphs. For instance, Gerard Ellis and Stephen Callaghan [12] have used conceptual scaling for improving the search in the generalization hierarchy of conceptual graphs. If the generalization order is represented as concept lattice, such a search might even be performed within a TOSCANA system (see [29]). Guy Mineau and Olivier Gerbé discuss in [24] context lattices in their definitional framework for contexts, based on Formal Concept Analysis.

As Formal Concept Analysis can be used for deriving and improving conceptual structures occuring in Conceptual Graphs, there may also be applications of Conceptual Graphs methods to Formal Concept Analysis. In particular, conceptual graphs could be very helpful in translating structural meaning of concept lattice into natural language to lead users of Formal Concept Analysis to a better understanding of possible interpretations. Another application would be to support knowledge acquisition for Conceptual Information Systems. In Conceptual Information Systems, the data are organized in a relational database. But if the data are only available in a less structured way (for instance from structured interviews), then Conceptual Graphs may support the formalization of the information, as they are close to natural language for being understood by the domain expert, and formal enough for being computationally tractable. Once the knowledge is represented in Conceptual Graphs, it can be transformed to formal contexts by using power context families (discussed in the next subsection).

\subsection{Integration: Unifying Systems of Logic}

An integration of developments in Conceptual Graphs and Formal Concept Analysis would mean to establish a common theoretical basis for the field of interest. An attractive field would be, as already indicated at the end of Section 3, knowledge-oriented systems of logic. In both disciplines, criticisms have been made about the inadequacy of mathematical logic concerning knowledge representation and processing. In [40], John Sowa lists a number of reasons why 
people who use a knowledge representation language may want to diverge from First Order Logic; those reasons are concerned with readability, computability, convenience, surprises, context dependence, and extended logics. All these issues have been actively explored in the Conceptual Graph community, and different approaches to them have been suggested, implemented, and published.

These activities might be combined with the recent development of "Contextual Logic" in Formal Concept Analysis (see [54], [55], [57], [26], [27]). Contextual Logic is understood as a mathematization of classical philosophical logic based on the elementary doctrines of concepts, judgments, and conclusions; Formal Concept Analysis yields the mathematization of the doctrine of concepts, and Conceptual Graphs is used for mathematizing the doctrines of judgments and conclusions. Contextual Logic is set-theoretically grounded on families of related contexts whose formal concepts allow a representation of the concepts and relations of conceptual graphs. Such representation of a conceptual graph is called a "Concept Graph" of the context family from which is is derived, and the family of related contexts is said to be a "Power Context Family". In [29] it is shown that the concept graphs of a power context family always form a lattice with respect to generalization, which can be represented as a concept lattice. Since power context families can always be derived from many-valued data contexts, the approach via Contextual Logic opens a large field of common applications for Conceptual Graphs and Formal Concept Analysis. This might stimulate to continue the process of integration of both disciplines and even further developments of logic systems.

\section{References}

1. ADAC: Gewußt wie - 10000 praktische Tips für alle Tage. ADAC Verlag, München 1993.

2. U. Andelfinger: Begriffliche Wissenssysteme aus pragmatisch-semiotischer Sicht. In: R. Wille, M. Zickwolff (eds.): Begriffliche Wissensverarbeitung-Grundfragen und Aufgaben. B.-I.-Wissenschaftsverlag, Mannheim 1994, 152-172.

3. A. Arnauld, P. Nicole: La logique ou l'art de penser. Amsterdam 1662.

4. F. Baader: Computing a minimal representation of the subsumption lattice of all conjunctions of concept defined in a terminology. In: Proc. Intl. KRUSE Symposium, August 11-13, 1995, UCSC, Santa Cruz 1995, 168-178.

5. H. Berg: Terminologische Begriffslogik. Diplomarbeit, TU Darmstadt 1997.

6. K. Biedermann: How triadic diagrams represent conceptual structures. LNAI 1257. Springer, Heidelberg 1997, 304-317.

7. R.J. Brachman: On the epistemological status of semantic networks. In: N.V. Findler (ed.): Associative networks: representation and use of knowledge by computers. Academic Press, New York 1979, 3-50 (reprinted in [8]).

8. R.J. Brachman, H.L. Levesque (eds.): Readings in Knowledge Represenation. Morgan Kaufmann, Los Altos 1985.

9. R. J. Brachman, D. L. McGuinness, P. F. Patel-Schneider, L. Alperin, L. A. Resnick, A. Borgida: Living With Classic: When and how to use a KL-ONE-like language. In: J. F. Sowa (ed.): Principles of Semantic Networks, Morgan Kaufmann, Los Altos 1991, 401-456. 
10. R. Davis, H. Schrobe, P. Szolovits: What is a knowledge representation? AI Magazine 14:1 (1993), 17-33.

11. Deutsches Institut für Normung: DIN 2330; Begriffe und Benennungen: Allgemeine Grundsätze. Beuth, Berlin-Köln 1979.

12. G. Ellis, S. Callaghan: Organization of knowledge using order factors. LNAI 1257, Springer, Heidelberg 1997, 342-356.

13. B. Ganter, S. O. Kuznetsov: Stepwise construction of the Dedekind-MacNeille Completion. LNAI 1453, Springer, Heidelberg 1998, 295-302.

14. B. Ganter, R. Wille: Formal Concept Analysis: Mathematical Foundations. Springer, Heidelberg 1999.

15. B. Groh, S. Strahringer, R. Wille: TOSCANA-Systems based on thesauri. LNAI 1453. Springer, Heidelberg 1998, 127-138.

16. N. Hartmann: Aristoteles und das Problem des Begriffs. In: Abh. Preuß. Akad. Wiss. Jg. 1939. Phil.-hist. Kl. Nr.5. Verlag Akad. Wiss., Berlin 1939.

17. M. Heidegger: Die Frage nach dem Ding. Niemeyer, Tübingen 1962.

18. I. Kant: Logic. Dover, New York 1988.

19. B. Kohler-Koch, F. Vogt: Normen und regelgeleitete internationale Kooperationen. In: G. Stumme and R. Wille (eds.): Begriffliche Wissensverarbeitung: Methoden und Anwendungen. Springer, Heidelberg (to appear)

20. W. Kollewe, C. Sander, R. Schmiede,R. Wille: TOSCANA als Instrument der bibliothekarischen Sacherschließung. In: H. Havekost and H.J. Wätjen (eds.): Aufbau und Erschließung begrifflicher Datenbanken. (BIS)-Verlag, Oldenburg 1995, 95-114.

21. F. Lehmann, R. Wille. A triadic approach to formal concept analysis. LNAI 954. Springer, Heidelberg 1995, 32-43.

22. P. Luksch, R. Wille: A mathematical model for conceptual knowledge systems. In: H.-H. Bock, P. Ihm (eds.): Classification, data analysis, and knowledge organization. Springer, Heidelberg 1991, 156-162

23. R. MacGregor: The evolving technology of classification-based knowledge representation systems. In: J. F. Sowa (ed.): Principles of semantic networks, Morgan Kaufmann, Los Altos 1991, 385-400.

24. G. W. Mineau, O. Gerbé: Contexts: A formal definition of worlds of assertions. LNAI 1257. Springer, Heidelberg 1997, 80-94.

25. W. Poguntke, R. Wille: Zur Restrukturierung der mathematischen Ordnungstheorie. In: A. M. Kempf, F. Wille: Mathematische Modellierung. McGraw-Hill, Hamburg 1986, 283-293.

26. S. Prediger: Simple concept graphs: a logic approach. LNAI 1453, Springer, Heidelberg 1998, 225-239.

27. S. Prediger: Kontextuelle Urteilslogik mit Begriffsgraphen. Ein Beitrag zur Restrukturierung der mathematischen Logik. Dissertation, TU Darmstadt. Shaker Verlag, Aachen 1998.

28. S. Prediger: Terminologische Merkmalslogik in der Formalen Begriffsanalyse. In: G. Stumme and R. Wille (eds.): Begriffliche Wissensverarbeitung: Methoden und Anwendungen. Springer, Heidelberg (to appear)

29. S. Prediger, R. Wille: The lattice of concept graphs of a relationally scaled context. Preprint, TU Darmstadt 1999. In this volume.

30. U. Priß: Relational concept analysis: semantic structures in dictionaries and lexical databases. Dissertation, TH Darmstadt 1996. Shaker Verlag, Aachen 1998. 
31. T. Rock, R. Wille: Ein TOSCANA-System zur Literatursuche. In: G. Stumme and R. Wille (eds.): Begriffliche Wissensverarbeitung: Methoden und Anwendungen. Springer, Heidelberg (to appear)

32. M. Roth-Hintz, M. Mieth, T. Wetter, S. Strahringer, B. Groh, R. Wille: Investigating SNOMED by Formal Concept Analysis. FB4-Preprint, TU Darmstadt 1998.

33. R. C. Schank, C. J. Rieger: Inference and the computer understanding of natural language, Artificial Intelligence Journal 5 (4) (1974), 373-412.

34. L. Searle, M. Keeler, J. Sowa, H. Delugagh, D. Lukose: Fulfilling Peirce's dream: conceptual structures and communities of inquiry. LNAI 1257. Springer, Heidelberg 1997, 1-11.

35. R.F. Simmons: Semantic networks: Their computation and use for understanding English sentences. In: R. C. Schank, K.M. Colby (eds.): Computer models of thought and language. Freeman, Can Francisco 1973, 63-113.

36. J. F. Sowa: Conceptual graphs for a data base interface. IBM Journal of Research and Development 20(1976), 336-357.

37. J. Sowa: Conceptual structures: information processing in mind and machine. Adison-Wesley, Reading 1984.

38. J. F. Sowa: Conceptual graphs summary. In: T. E. Nagle, J. A. Nagle, L. L. Gerholz, P. W. Eklund (eds.): Conceptual Structures: Current Research and Practice. Ellis Horwood 1992, 3-51.

39. J. F. Sowa: Knowledge representation: logical, philosophical, and computational foundations. PWS Publishing Co., Boston (to appear)

40. J. F. Sowa: Conceptual Graph standard and extension. LNAI 1453. Springer, Heidelberg 1998, 3-14.

41. N. Spangenberg, K. E. Wolff: Comparison between principal component analysis and formal concept analysis of repertory grids. In: W. Lex (ed.): Arbeitstagung Begriffsanalyse und Künstliche Intelligenz, Informatik-Bericht 89/3. TU Clausthal, 1991, 127-134.

42. S. Strahringer, R. Wille: Towards a structure theory for ordinal data. In: M. Schader (ed.): Analyzing and Modeling Data and Knowledge. Springer, Heidelberg 1992, 129-139.

43. G. Stumme: Knowledge acquisition by distributive concept exploration. Suppl. Proc. 3rd ICCS, Santa Cruz, CA, USA, 1995, 98-111.

44. G. Stumme: Local scaling in conceptual data systems. LNAI 1115. Springer, Heidelberg 1996, 308-320.

45. G. Stumme: Exploration tools in Formal Concept Analysis. In: Ordinal and Symbolic Data Analysis. Studies in Classification, Data Analysis, and Knowledge Organization 8, Springer, Heidelberg 1996, 31-44.

46. G. Stumme: Concept exploration - a tool for creating and exploring conceptual hierarchies. LNAI 1257. Springer, Heidelberg 1997, 318-331.

47. F. Vogt: Formale Begriffsanalyse mit $C++$ : Datenstrukturen und Algorithmen. Springer, Heidelberg 1996.

48. Stiftung Warentest: Kaffeemaschinen mit Warmhaltekannen: Wärme okay, Geschmack ade. Test 12/98, 71-75.

49. R. Wille: Restructuring lattice theory: an approach based on hierarchies of concepts. In: I. Rival (ed.): Ordered sets. Reidel, Dordrecht, Boston 1982, 445-470.

50. R. Wille: Knowledge acquisition by methods of formal concept analysis. In: E. Diday (ed.): Data analysis, learning symbolic and numeric knowledge. Nova Science Publishers, New York-Budapest 1989, 365-380. 
51. R. Wille: Plädoyer für eine philosophische Grundlegung der Begrifflichen Wissensverarbeitung. In: R. Wille, M. Zickwolff (eds.): Begriffliche Wissensverarbeitung: Grundfragen und Aufgaben. B.I.-Wissenschaftsverlag, Mannheim 1994, 11-25.

52. R. Wille: Begriffsdenken: Von der griechischen Philosophie bis zur Künstlichen Intelligenz heute. In: Dilthey-Kastanie. Ludwig-Georgs-Gymnasium, Darmstadt 1995, 77-109.

53. R. Wille: Conceptual structures of multicontexts. LNAI 1115. Springer, Heidelberg 1996, 23-39.

54. R. Wille: Restructuring mathematical logic: an approach based on Peirce's pragmatism. In: A. Ursini, P. Agliano (eds.): Logic and Algebra. Marcel Dekker, New York 1996, 267-281.

55. R. Wille: Conceptual Graphs and Formal Concept Analysis. LNAI 1257. Springer, Heidelberg 1997, 290-303.

56. R. Wille: Conceptual landscapes of knowledge: a pragmatic paradigm for knowledge processing. In: G. Mineau and A. Fall (eds.): Proc. 2nd Intl. KRUSE. Simon Fraser University, Vancouver 1997, 2-13.

57. R. Wille: Triadic Concept Graphs. LNAI 1453, Springer, Heidelberg 1998, 194-208.

58. R. Wille: Bildung und Mathematik. FB4-Preprint Nr. 2005, TU Darmstadt 1998.

59. W. Zadrozny: Logical dimensions of some graph formalisms. In: J. F. Sowa (ed.): Principles of semantic networks. Morgan Kaufmann, Los Altos 1991, 363-380. 